\title{
Quellmonitoring zur optimierten Quellwassernutzung
}

\author{
Benedikt-Johannes Schmidt · Ernest Mayr · Christoph Schönher · Marija Zunabovic-Pichler · Reinhard Perfler
}

Online publiziert: 11. April 2017

(c) Der/die Autor(en) 2017. Dieser Artikel ist eine Open-Access-Publikation.

Zusammenfassung Im Rahmen eines Forschungsprojekts wurde bei einzelnen Quellfassungen eine kontinuierliche Überwachung zur Erfassung von Veränderungen der Trinkwassereigenschaften aufgrund hydrologischer Ereignisse durchgeführt. Die Quellfassungen wurden dabei mit einer kontinuierlichen Erfassung von Schüttung (in 1/s mittels Messwehr und Wasserstand bzw. Wasserzähler), Wassertemperatur $\left(\right.$ in $\left.{ }^{\circ} \mathrm{C}\right)$, elektrischer Leitfähigkeit $(\mu \mathrm{S} / \mathrm{cm})$, Trübung (FTU), spektralem Absorptionskoeffizient (SAK254) (Abs/m), gelöstem organischem Kohlenstoff (DOC $\mathrm{mg} / \mathrm{l}$ ) und Transmission (\%) jeweils mittels 10-Minuten- bzw. Stundenwerten überwacht. Zusätzlich wurde zur Erfassung von unmittelbaren Niederschlagsereignissen im Fassungsgebiet eine Niederschlagsmessung (Zeit und Menge) eingesetzt und es wurden vergleichende Laboranalysen durchgeführt. Im Rahmen dieser Überwachung wurde zusätzlich die Anwendung der eingesetzten Messinstrumente an Extremstandorten (z. B. kein Stromanschluss) evaluiert.

Für den Wasserversorger können durch die Anwendung einer über eine gewisse Zeit durchgeführten kontinuierlichen Quellüberwachung Vorteile für die Einschätzung besonderer Betriebssituationen durch Kenntnis der Beeinflussung und des Ausmaßes entstehen, um eine optimierte Quellnutzung aufzubauen. Sind die quellspezifischen Wirkungen auf Basis eines Quellmonitorings bekannt (Prozessverständnis), kann die Quellnutzung oft mit sehr einfachen Maßnahmen, wie kurzfristiges Ausleiten des nativen Wassers oder Einsatz einer zeitlich begrenz-

DI B.-J. Schmidt $(\triangle)$.

DI E. Mayr · C. Schönher ·

DI Dr. M. Zunabovic-Pichler .

Priv.-Doz. DI Dr. R. Perfler

Institut für Siedlungswasserbau, Industriewasserwirtschaft und

Gewässerschutz, Universität für Bodenkultur Wien,

Muthgasse 18, 1190 Wien, Österreich benedikt.schmidt@boku.ac.at ten Desinfektionsmaßnahme, optimiert werden.

Durch die Anwendung von Messinstrumenten mit geringer Stromaufnahme und Akkusystemen konnte auch an Extremstandorten eine Überwachung über mehrere Monate durchgeführt werden.

Aufbauend auf dem durchgeführten Quellmonitoring konnten Anforderungen an eine Messstation definiert werden.

\section{Monitoring to optimize well-water use}

Abstract In the context of a research project, individual wells were continuously monitored to identify and measure changes in drinking-water quality in response to hydrological events. Specifically, the criteria flow rate (in 1/s using a measuring weir and water level, or water flow meter), water temperature (in ${ }^{\circ} \mathrm{C}$ ), electrical conductivity $(\mu \mathrm{S} / \mathrm{cm})$, turbidity (FTU), spectral absorption coefficient (SAC 254), dissolved organic carbon (DOC $\mathrm{mg} / \mathrm{l}$ ) and transmission (\%) were continuously monitored at 10 minute or hourly intervals. In addition, the times and volumes of precipitation events were recorded, and comparative laboratory analyses were conducted. Lastly, the monitoring equipment's suitability for use at extreme locations (e.g. those with no electrical power outlets) was evaluated.

For water providers, continuous monitoring could help to assess special operating situations, providing insights into their scope of influence and helping promote optimized well use. Once well monitoring has been used to determine the well-specific effects, the usage of the well can often be optimized with the help of simple measures, e.g. temporarily diverting the native water or employing a short-term disinfectant.

By using measuring equipment with low power requirements and rechargeable battery systems, it was also possible to conduct monitoring over several months at extreme locations. Further, the results of the well-monitoring efforts were used to define a set of criteria for monitoring stations.

\section{Einleitung und Hintergrund}

In Österreich werden ca. 50 \% der zentral versorgten Einwohner mit Quellwasser aus ca. 2.600 Quellfassungen versorgt (ÖVGW 2013). Die einwandfreie Wasserqualität wird mittels Untersuchungen lt. Trinkwasserverordnung nachgewiesen. In der Regel werden bei den einzelnen Quellfassungen zusätzlich die Quellschüttung und ausgewählte Qualitätsparameter wie Temperatur und elektrische Leitfähigkeit monatlich erfasst. Anhand dieser Daten können längerfristige Trends bei der Wasserqualität und der Menge erfasst werden. Mit diesen Datenreihen von Einzelmessungen kann jedoch kaum eine Aussage über mögliche kurzfristige Veränderungen der Quellschüttung und/oder Wasserqualität getroffen werden. Diese Varianz könnte z. B. von meteorologisch kleinräumigen Ereignissen wie Starkregenniederschläge, Schneeschmelze etc., gerade an weniger geschützten Quellstandorten wie bei Karst-, Kluftoder zu seicht gefassten Quellen, ausgelöst werden. Aber auch anthropogene Einflüsse sind möglich (Treskatis und Tauchmann 2013). Kurzfristige Ereignisse, die zudem auch nur an wenigen Tagen im Jahr auftreten können, sind oftmals nur durch eine kontinuierliche Datenerfassung zu erkennen. Sind die quellspezifischen Wirkungen auf Basis eines Quellmonitorings bekannt (Prozessverständnis), kann die Quellnutzung oft mit sehr einfachen Maßnahmen, wie kurzfristiges Ausleiten des nativen Wassers oder Einsatz einer zeitlich begrenzten Desinfektionsmaßnahme, optimiert werden.

Die kontinuierliche Erfassung der Wasserqualität eines Quellmonitorings bildet damit die relevante Grundlage, um eine optimierte Quellnutzung oder das Setzen von möglichen Verbesserungsmaßnahmen abzuleiten. Grundsätzlich sollten mit einem zeitlich begrenzten Quellmonitoring einzelne leicht $\mathrm{zu}$ erfassende Steuerungspara- 


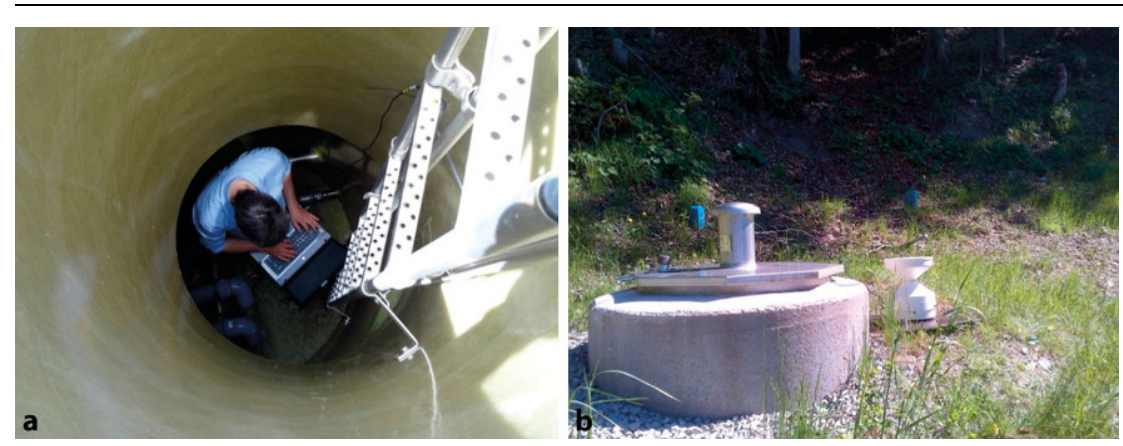

Abb. 1 Auslesen der Messinstrumente (links) sowie Aufbau einer Niederschlagsmessung bei einem Quellsammelschacht (rechts)

meter gefunden werden, die dann wieder vereinfacht eine optimierte Quellnutzung ermöglichen.

Für den Wasserversorger können durch die Anwendung einer, über eine gewisse Zeit durchgeführten kontinuierlichen Quellüberwachung, folgende Vorteile für die Einschätzung besonderer Betriebssituationen entstehen:

- Kenntnis der (zeitlich begrenzten) Beeinflussung der Trinkwassereigenschaften bei einem hydrologischen Ereignis (z. B. Starkregenereignis, Trockenperioden, Starkregen nach Trockenperiode, Schneeschmelze).

- Ausmaß und zeitliche Ausdehnung der möglichen Beeinflussung der Trinkwassereigenschaften mittels möglichst aussagekräftiger physikalisch-chemischer Parameter (mögliche mikrobiologische Beeinflussung sollte bei bestimmten Beeinflussungen zusätzlich im Einzelnen untersucht werden).

- Ermittlung von einfach zu erfassenden Ersatzparametern (z. B. elektrische Leitfähigkeit) zur Erfassung hydrologischer Ereignisse und Schaffung von Ausleitungs- und Einspeisekriterien - nach vorheriger Bewertung mittels umfassender kontinuierlicher Überwachung und Risikominimierung potentieller mikrobiologischer Eintragungspfade.

\section{Zielsetzung}

Ganz allgemein sollen mithilfe eines gezielt eingesetzten Quellmonitorings Informationen über kurzfristige Veränderungen von Menge und Qualität bei Ereignissen als strategische Entscheidungsgrundlage gesammelt und einzelne (automatisierte) Steuerungsparameter abgeleitet werden. Diese Entscheidungen können das Ausleiten von Quellen bei veränderten Trink- wassereigenschaften oder der zeitlich begrenzte Einsatz einer Desinfektionsmaßnahme darstellen.

In dieser Arbeit lag die Zielsetzung auf dem Aufbau einer Teststellung eines Quellmonitorings an unterschiedlichen Extremstandorten mit dezentraler Stromversorgung zur Optimierung der Anwendung. Weiters sollten die Datensammlung und Übertragung sowie die Umsetzung einer zeitnahen Auswertung als Entscheidungsgrundlage für unterschiedliche Betriebszustände entwickelt und die Anforderungen an eine Quellmessstation definiert werden.

Es war nicht Ziel dieser Arbeit, unterschiedliche Messinstrumente für den Einsatz bei einem Quellmonitoring zu testen oder zu beurteilen, sondern es wurden - aufgrund der leichten Verfügbarkeit - handelsübliche Messinstrumente (System) eingesetzt.

Generell ist der Informationsgewinn aus der detaillierteren Beobachtung nicht nur in der Verbesserung bei kurzfristigen Veränderungen (Menge und Qualität) zu sehen, sondern hilft auch langfristig als Grundlage für strategische Entscheidungen bei der Quellnutzung.

\section{Durchführung eines Quellmonitorings}

Im Rahmen unterschiedlicher Fallstudien wurden Messreihen aufgezeichnet und entsprechend aufbereitet. Hierbei zeigten sich auch die Schwierigkeiten bei der Installation und Wartung (siehe Abb. 1): einerseits die Erreichbarkeit und Ausstattungsmerkmale der exponierten Versuchsstandorte und zum anderen die beengten Platzverhältnisse.

Für die Untersuchungen wurden unterschiedlichen Quellfassungen mit Schüttungsschwankungen bei Schnee- schmelze und starken Regenereignissen sowie Unterschiede bei einzelnen Fassungssträngen ausgewählt. Weiters wurden an Einzelterminen auch mehrere einzelne Quellen in einem Einzugsgebiet untersucht, um mögliche Unterschiede der Quellfassungen bei stabilen hydrologischen Verhältnissen zu erfassen.

Zur Untersuchung des Einflusses von Schüttungsschwankungen auf die Wasserqualität wurden Untersuchungen mit kontinuierlich aufzeichnenden Messgeräten durchgeführt. Bei keiner der untersuchten Quellen war ein Stromanschluss vorhanden, die Versorgung der Messstation wurde mittels Batterien/Akkus durchgeführt.

Für das Quellmonitoring wurden dafür unterschiedliche Messgeräte, zeitweise überlappend, eingesetzt:

- Niederschlagsmessung für Lufttemperatur $\left({ }^{\circ} \mathrm{C}\right)$ und Regenspende $(\mathrm{mm} / \mathrm{h})$,

- Wasserstandmessung für Umrechnung auf Schüttung in l/s über Wehrformel,

- Schüttungsmessung über Durchflussmessung,

- Wasserqualität mithilfe von Temperatur $\left({ }^{\circ} \mathrm{C}\right)$ und elektr. Leitfähigkeit $(\mu \mathrm{S} / \mathrm{cm})$ sowie Trübung (FTU), SAK $254(\mathrm{Abs} / \mathrm{m})$, gelöster organischer Kohlenstoff - DOC (mg/l) und UV-Transmission (\%) bei $10 \mathrm{~cm}$ Schichtdicke,

- Probenehmer für Einzelprobenahmen,

- ereignisbasierte Probenahme durch Betriebspersonal (mehrmals täglich).

Zur Überprüfung der kontinuierlichen Datenerfassung wurden Handmessungen von Vor-Ort-Parametern sowie Probenahmen für Laboranalysen durchgeführt. Die einzelnen Handmessungen konnten nicht immer den tatsächlichen Zeitpunkten (Uhrzeit) zugeordnet werden. Wurde nur das Datum aufgezeichnet, werden die Werte um 0:00 Uhr angezeigt, was zu einzelnen Abweichungen zwischen Handmessung und kontinuierlicher Aufzeichnung führen kann.

\section{Ergebnisse Quellmonitoring}

\subsection{Fallstudie 1}

Die Niederschlagsmessung und die Aufzeichnung der Lufttemperatur wurden nicht über den gesamten Beobachtungszeitraum durchgeführt. Die 


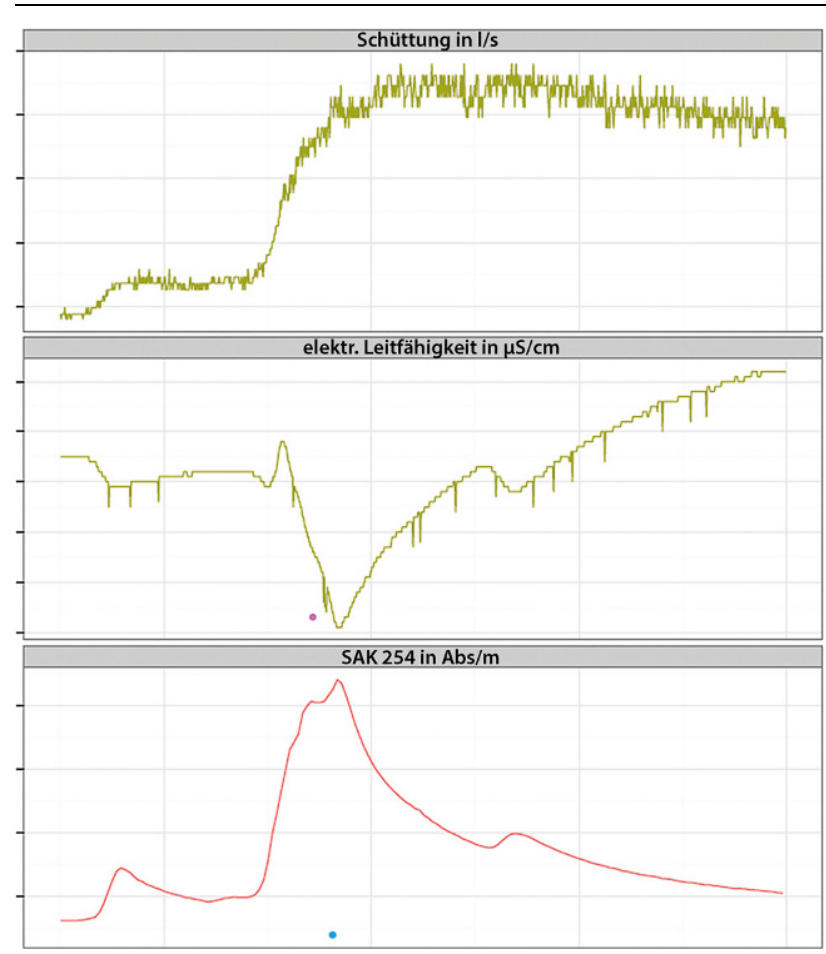

Abb. 2 Kontinuierliche Datenerfassung der einzelnen Parameter (anonymisierte Werte) bei einer Quellfassung und vergleich mit Handmessungen (Punkte)

Schüttung wurde mittels Wasserstandsaufzeichnung über die Wehrformel berechnet. Bei starken Regenereignissen wurde ein deutlicher Anstieg (Verdoppelung bzw. das 2,5-Fache) der Schüttung in einem Zeitraum über mehrere Stunden beobachtet. Die Erfassung der Verzögerungszeit $\mathrm{zu}$ den zugehörigen sehr kleinräumigen Regenereignissen, konnte aufgrund der zu diesen Zeitpunkten fehlenden Niederschlagsmessung direkt im Fassungsbereich, nicht durchgeführt werden. Bei einem starken Anstieg der Schüttung konnte in der vergleichsweisen kühlen Jahreszeit im März, eine Verringerung der Wassertemperatur und in der warmen Jahreszeit im Mai, ein leichter Anstieg beobachtet werden. Bei den starken Regenereignissen (Abb. 2) wurde ein kurzfristiger und starker Abfall der elektrischen Leitfähigkeit um ca. 30 bis $40 \mu \mathrm{S} / \mathrm{cm}$ beobachtet. Bei geringeren Regenereignissen wurde lediglich ein leichtes, kurzfristiges Abfallen festgestellt. Über den gesamten Beobachtungszeitraum konnte keine wesentliche Veränderung der Trübung beobachtet werden. Mit der Messung des Summenparameters Spektraler Absorptionskoeffizient bei $254 \mathrm{~nm}$ (SAK 254) kann ohne aufwendige Einzelstoffana- lytik eine Aussage über die Belastung eines Wassers mit (UV-absorbierenden) organischen Stoffen gemacht werden. Bei starken Regenereignissen und einem hohen Schüttungsanstieg konnte auch ein sehr starker, aber kurzfristiger Anstieg des SAK 254 beobachtet werden, was auf einen kurzzeitigen Eintrag von organischen Stoffen schließen lässt. Der gelöste organische Kohlenstoff (DOC) setzt sich als Summenparameter aus tausenden Einzelsubstanzen zusammen, die - außer der Gemeinsamkeit der organischen Verbindung - ganz unterschiedliche, funktionelle chemische Gruppen aufweisen. Wie auch der SAK 254 steigt der DOC mit Änderung der Schüttung kurzzeitig sehr stark an. Die UV-Durchlässigkeit enthält nach ÖNORM M 5873-1 (ÖNORM 2001) die Einflüsse der Streuung und der Absorption des durchlaufenden Mediums und dient daher als wichtige Größe zur Auslegung einer UVDesinfektion. Die UV-Durchlässigkeit hat auf die Wirkung des UV-Desinfektionsverfahrens entscheidenden Einfluss und wird durch gewisse Stoffe (z. B. Huminsäuren, Eisen- und Manganverbindungen) bestimmt, die im Wasser in gelöster Form oder als suspendierte Teilchen enthalten sein können. Im Ge- gensatz zur UV-Durchlässigkeit, wird die UV-Transmission an der gefilterten Probe bestimmt und umfasst somit nur die Absorption durch gelöste Stoffe und nicht auch die Streuung an suspendierten Partikeln. Mit Anstieg des organischen Materials bei starken Regenereignissen (SAK 254, DOC) wurde gleichzeitig der Abfall der UV-Transmission bei $10 \mathrm{~cm}$ Schichtdicke auf einen Minimumwert unter $10 \%$ beobachtet.

Bei den Untersuchungen über mehrere Monate konnten sehr stabile Verhältnisse, aber jedoch auch kurzzeitige „Stoßbelastungen“ von organischem Material beobachtet werden, die nach einem Schüttungsanstieg in wenigen Tagen wieder auf das Ausgangsniveau zurückgingen. Untersuchungen, um den Zusammenhang zwischen Anstieg des organischen Materials (DOC und SAK 254) und einem möglichen Anstieg einer mikrobiologischen Belastung zu erfassen, wurden bei dieser Fallstudie nicht durchgeführt. Höhere Anteile von organischem Material können jedoch ein Potenzial für das mikrobiologische Wachstum darstellen (siehe Fallstudie 3). Bei einzelnen Probenahmen konnten keine mikrobiologischen Standardparameter nachgewiesen werden.

Für die weitere Vorgehensweise wurden folgende Punkte vorgeschlagen:

- Kontinuierliche Überwachung der Quelle mit einer Sonde zur Erfassung von Wasserstand (Schüttung), Temperatur und elektrischer Leitfähigkeit.

- Durchführung einer verdichteten Probenahme bei einem Regenereignis mit dem Ziel, einen Zusammenhang zwischen Veränderung der elektrischen Leitfähigkeit als Prozessparameter und Nachweis einer möglichen mikrobiologischen Kontamination herzustellen.

Bei einem möglichen Zusammenhang könnte eine zeitlich begrenzte Desinfektionsmaßnahme bzw. das Ausleiten des Quellwassers mittels des einfach zu messenden Steuerungsparameters der elektrischen Leitfähigkeit gesteuert werden. Der Einbau einer kontinuierlich betriebenen Desinfektionsmaßnahme scheint aufgrund der konstanten mikrobiologischen Wasserqualität nicht zweckdienlich.

\subsection{Fallstudie 2}

Die Niederschlagsmessung und die Aufzeichnung der Lufttemperatur wurden 


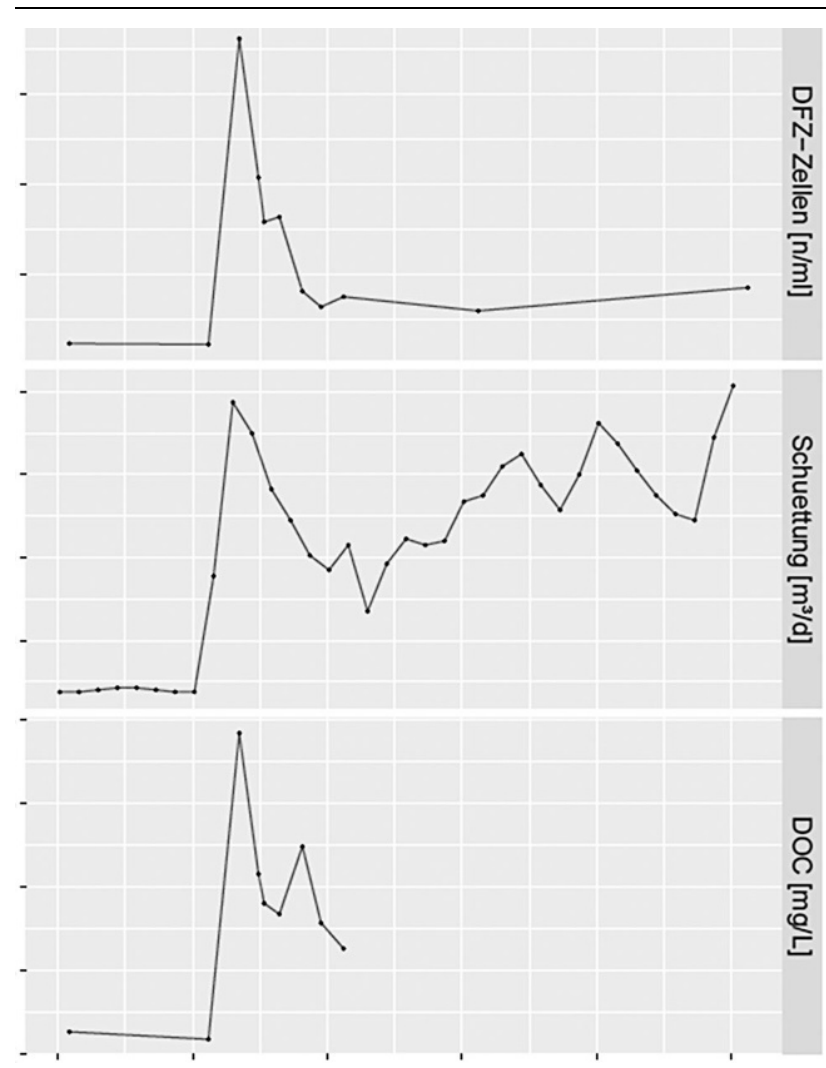

Abb. 3 Vergleich der Totalzellzahl (Durchflusszytometrie) mit der Schüttung und dem gelösten organischen Kohlenstoff bei einer Quellwasserversorgung (Rohwasser)

kontinuierlich durchgeführt. Kontinuierliche, längere Regenereignisse zeigen die größte Auswirkung auf die Schüttung, während sehr kurze, aber starke Regenereignisse bis $30 \mathrm{~mm} / \mathrm{h}$ nur einen sehr geringen Einfluss zeigen. Dies könnte auch durch die Vegetation vor Ort (Wiese) und/oder die vorhandene Sonnenscheindauer (Verdunstung) in diesem Zeitraum beeinflusst sein. Die Schüttung wurde mittels eingebauter Durchflussmessungen für die unterschiedlichen Fassungsstränge einzeln erfasst. Bis auf einen Fassungsstrang zeigen alle Fassungsstränge einen konstanten Schüttungsverlauf im Beobachtungszeitraum. Die Wassertemperatur bleibt im gesamten Beobachtungszeitraum konstant. Nach einem längeren Regenereignis (Anstieg der Schüttung in einem Fassungsstrang), wurde ein kurzfristiger starker Abfall der elektrischen Leitfähigkeit um ca. $30 \mu \mathrm{S} / \mathrm{cm}$ über den Zeitraum des Schüttungsanstiegs beobachtet. Über den gesamten Beobachtungszeitraum konnte keine wesentliche Veränderung der Trübungswerte $<0,1$ NTU beobachtet werden. Bei dem Regenereignis sowie auch beim Schüt- tungsanstieg im Fassungsstrang konnte ein kurzfristiger Abfall des SAK 254, DOC- und Nitratgehalts beobachtet werden, was zusätzlich auf eine Verdünnung des Grundwassers mit weniger mineralisiertem, oberflächennah abgeflossenem Wasser hinweist.

Ausschlaggebend ist jedoch nicht die Höhe des Niederschlags, als vielmehr die Dauer eines Niederschlagsereignisses, vergleichbar mit einem „Filterdurchbruch" bei Filtern. Nach diesen intensiven Regenereignissen verringert sich auch die Schüttung im Fassungsstrang nach kurzer Zeit auf ihr Ausgangsniveau, was auf einen direkten Zusammenhang schließen lässt.

Auf Basis des Quellmonitorings wurde als Maßnahme eine Sanierung des Fassungsstrangs (Neufassung) vorgeschlagen.

\subsection{Fallbeispiel 3}

In einem vom Bundesministerium für Land- und Forstwirtschaft, Umwelt und Wasserwirtschaft (BMLFUW 2017) und von der Österreichischen Vereinigung für das Gas- und Wasserfach (ÖVGW) geförderten Projekts zur mikrobiologischen Charakterisierung von Einflussfaktoren und Zustandsveränderungen bei der Wasserversorgung (Ressourcen) anhand der Durchflusszytometrie konnten bei ausgewählten Quellwasserversorgungen systematische Untersuchungen des Zusammenhangs von unterschiedlichen mikrobiologischen und Standardparametern durchgeführt werden. Unter Zytometrie versteht man das Zählen von Zellen beispielsweise mittels automatisierter Epifluoreszenzmikroskopie, Coulter Counter oder der Durchflusszytometrie. Das Prinzip der durchflusszytometrischen Methode (DFZ) basiert auf der Färbung von Zellen in einer Probe (z. B. Wasser) mit einem Fluoreszenzfarbstoff oder je nach Fragestellung mit einem Farbstoffgemisch. Wird die gefärbte Probe nun dem Durchflusszytometer zugeführt, werden die Zellen hydrodynamisch fokussiert und von einem Laserstrahl erfasst, wodurch jede einzelne Zelle zu einer Lichtstreuung und Fluoreszenz führt. Diese Signale werden über Filter und Spiegel auf Detektoren geleitet und erfasst. Dieser Prozess wird tausend Mal pro Sekunde wiederholt, wodurch mehrere tausend Zellen in kürzester Zeit mit hoher Präzision detektiert werden. Die Bestimmung der Totalzellzahl (TZZ) in einer Wasserprobe (pro $\mathrm{ml}$ ) ist ebenso möglich wie die Erfassung der Vitalität von Zellen (lebend/tot) oder der spezifische Nachweis von Krankheitserregern (z. B. Legionellen) durch Markierung mit beispielsweise Antikörpern. Die Ergebnisse einer TZZ sind mit der "Gesamtkeimzahl“ nicht zu vergleichen, da es sich bei dieser um die Anzahl der am Nährmedium kultivierbaren Bakterienzellen handelt. Eine kultivierungsabhängige Methode benötigt in etwa 1 bis 3 Tage, während die Bestimmung der TZZ etwa 15 Minuten erfordert. Mittlerweile werden auch Online-Geräte zur Erfassung der Totalzellzahl am Markt angeboten.

In Abb. 3 ist der Vergleich der Totalzellzahl (Durchflusszytometrie) mit der Schüttung und dem gelösten organischen Kohlenstoff bei einer Quellwasserversorgung (Rohwasser) dargestellt. Der Zusammenhang zwischen Veränderung der Totalzellzahl bei Anstieg der Schüttung bei diesem außergewöhnlichen Extremereignis ist deutlich $\mathrm{zu}$ erkennen.

Da die Durchflusszytometrie eine sehr sensitive Methode zur Erfassung von Veränderungen der mikrobiolo- 
- Hauptgruppe - Ausreißer

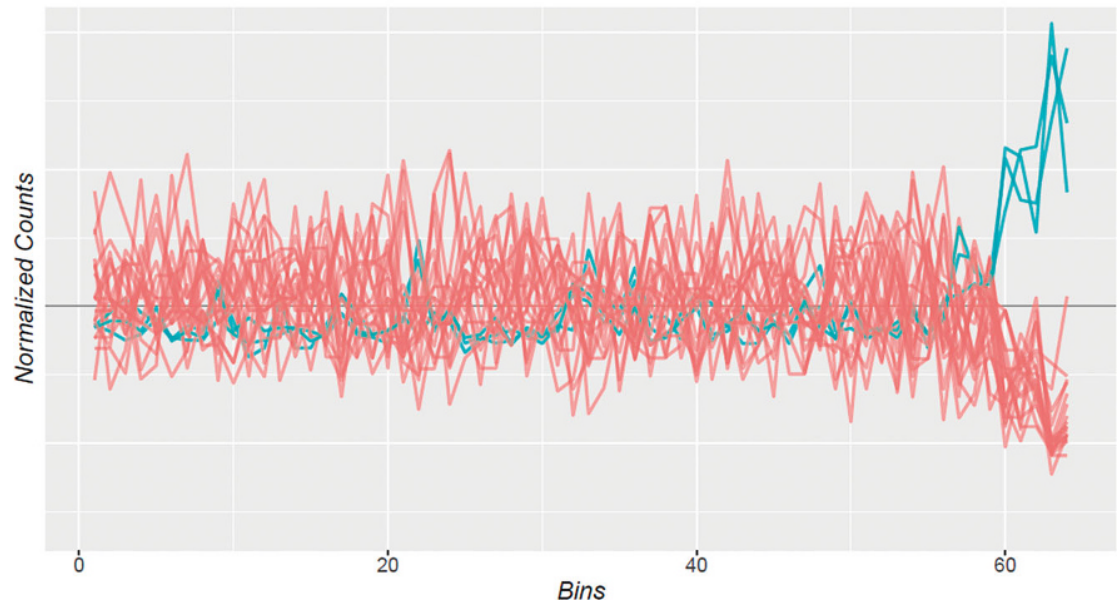

Abb. 4 Fingerprintmodell unterschiedlicher Totalzellzahlen auf Basis ihrer Fluoreszenzeigenschaften für 30 unterschiedliche Quellen in einem Einzugsgebiet (rot) und 3 Quellen mit Abweichung davon (blau, Ausreißer)

gischen Zellzahlen darstellt, wurde sie auch dafür angewendet, um bei einer einmaligen Untersuchung von 30 Quellfassungen in einem einzelnen Einzugsgebiet mögliche Unterschiede zwischen einzelnen Quellen festzustellen. Auf Basis dieser Voruntersuchung sollten einzelne Quellen für ein weiteres Quellmonitoring ausgewählt werden. In Abb. 4 wurde ein Fingerprintmodell der Zellzahleigenschaften erstellt. Auf der $\mathrm{x}$-Achse sind dabei die normalisierten Zellzahlen einzelner Abschnitte und auf der y-Achse die zugehörigen Fluoreszenzeigenschaften aufgetragen. Deutlich zu erkennen ist, dass sich drei Quellen signifikant von den anderen Quellen unterscheiden (blau). Für diese wurden weiterführende Untersuchungen im Quellmonitoring durchgeführt.

\section{Anforderungen an eine Messstation}

Für die Anwendung eines Quellmonitorings mussten zuerst unterschiedliche Messsysteme kombiniert werden, um die notwendigen Messgrößen zu erfassen. Daraus erwachsen jedoch verschiedene Probleme:

- Jedes Messgerät hat eine eigene Speicherfunktion, die separat ausgelesen werden muss. Durch die Ungenauigkeit der integrierten Zeiterfassung kann eine Zeitdrift entstehen.

- Unterschiedliche Messintervalle erschweren es, einen gleichzeitigen Messzeitpunkt festzulegen. So kann es vorkommen, dass ein Messgerät als Intervalle 5, 10 oder 30 Minuten festlegt, ein anderes jedoch nur Viertelstundenwerte und Vielfache davon zulässt. Es gilt dann abzuwägen, längere gemeinsame Messintervalle festzulegen, oder unterschiedlich detaillierte Messreihen in Kauf zu nehmen.

- Jedes Messgerät speichert den Zeitstempel in einer unterschiedlichen Detaillierung ab. Messwerte werden teilweise auf volle Minuten gerundet, teils wird die exakte Sekunde mitprotokolliert. Für eine Zusammenführung der Daten muss ein einheitliches Format definiert werden.

- Die Stromversorgung der Geräte ist unterschiedlich, wodurch es keine einheitliche Laufzeit gibt. Teilweise besteht keine Möglichkeit, die Akkus von Geräten, die für die Anwendung optimal wären, z. B. durch Anschluss an externe (dezentrale) Stromversorgung, zu ersetzen.

- Quellstandorte liegen normalerweise außerhalb der Infrastruktureinrichtungen, weshalb die Stromversorgung durch langlebige Akkus erfolgen muss; optimal ist eine entsprechend dimensionierte Photovoltaikanlage, die jedoch nicht an jedem Standort über den Großteil des Jahres einsetzbar ist.

- Bei den meisten Messgeräten ist es nicht möglich, einen Trigger festzulegen, um ein Messintervall $\mathrm{zu}$ verdichten (z. B. Regenereignis oder Messwert außerhalb der Schwankungsbreite).

- Um die gewonnenen Daten auch für zeitnahe Steuerungsentscheidungen verwenden zu können, ist zusätzlich eine Internetanbindung notwendig.

Daraus ergibt sich, dass eine einfache, stromsparende Plattform fehlt, die verschiedene Messsysteme vereint und zusätzlich auch eine schnelle Bereitstellung der Daten ermöglicht.

Ebenfalls festzulegen ist, dass die Quellmessstation nur als Informationsquelle für ein größeres System dienen soll, das von einer Minimumüberwachung hochgefahren werden kann.

\section{Umsetzung Quellmessstation}

Derzeit wird am Institut für Siedlungswasserbau, Industriewasserwirtschaft und Gewässerschutz der Universität für Bodenkultur Wien eine integrierte Messstation weiterentwickelt, die folgende Funktionen bieten soll:

- Aufzeichnung verschiedener Messwerte und Übertragung auf einen Server.

- Anpassung des Messintervalls automatisiert bei Regenereignissen oder Veränderung von Messwerten über einen festgelegten Vertrauensbereich.

- Definition von Alarmgrenzwerten und Versand von SMS oder Schaltung von Relais (Ansteuerung Probenehmer, gegebenenfalls Quellausleitung etc.).

Als größte Herausforderung für Quellmessstationen ist sicherlich die exponierte Lage zu bezeichnen. Neben der oft schwierigen Zugänglichkeit in entlegenen Gebieten sind auch fehlender Stromanschluss und schlechte Internetanbindung kennzeichnend für diese Messstationen. Fehlender Strom muss teilweise mit Akkus überbrückt werden, die gegebenenfalls durch Solarenergie aufgeladen werden können. Dadurch ergibt sich, dass Messsysteme nur möglichst geringen Strombedarf haben dürfen; PC-basierte Systeme scheiden daher im Normalfall aus. Ebenfalls können Möglichkeiten mit kleinen Turbinen oder Windkraft in Betracht gezogen werden.

Mangelhafte Internetanbindung kann über verschiedene Wege gelöst werden. Für die erste Phase, in der es um reines Monitoring und anschließende Datenauswertung geht, kann auch auf die direkte Übertragung verzichtet werden und eine reine Speicherung auf SDKarte erfolgen. Die Auswertung erfolgt dann beispielsweise halbjährlich. Wenn 


\begin{tabular}{|c|c|c|}
\hline & Messstation als Client & Messstation als Server \\
\hline Funktionsweise & $\begin{array}{l}\text { Die Messstation startet in regelmäßigen Abständen eine Übertra- } \\
\text { gung auf einen Server und lädt sich auch gegeben falls Konfigurati- } \\
\text { onsfiles herunter. }\end{array}$ & $\begin{array}{l}\text { Die Messstation wartet auf eine Anfrage durch ein anderes Gerät } \\
\text { und beantwortet diese direkt. }\end{array}$ \\
\hline Vorteile & Sparsamer im Stromverbrauch & Konfiguration kann jederzeit geändert werden \\
\hline Nachteile & $\begin{array}{l}\text { Messstation kann nicht jederzeit erreicht werden, Konfigurationen } \\
\text { nur über Konfigurationsdateien möglich, die der Client herunterlädt. }\end{array}$ & $\begin{array}{l}\text { Server benötigt eine feste IP-Adresse, um erreichbar zu sein. } \\
\text { Durch die ständige Verfügbarkeit erhöhter Stromverbrauch. }\end{array}$ \\
\hline
\end{tabular}

Tab. 2 Vergleich verschiedener Plattformen

\begin{tabular}{l|l|l|}
\hline CPU Takt in MHz & RaspberryPI & Arduino \\
\hline $\begin{array}{l}\text { Stromverbrauch (Watt laut ELEKTRONIK-KOMPENDI- } \\
\text { UM } 2017 \text { bzW. ATMEL 2017) }\end{array}$ & $700-1200$ & $16-20$ \\
\hline $\begin{array}{l}\text { Industriedesign } \\
\text { Verfügbare Bibliotheken }\end{array}$ & $\begin{array}{l}\text { Nein } \\
\text { Viele Programme aus Linux direkt verwendbar }\end{array}$ & $\begin{array}{l}\text { Nein } \\
\text { Große Community mit vielen verfügbaren Bibliothe- } \\
\text { ken, die aber teilweise nicht kompatibel zueinan- } \\
\text { der sind }\end{array}$ \\
\hline Rechenleistung & $\begin{array}{l}\text { Gut, auch für umfangreichere Aufgaben geeignet, } \\
\text { Multitasking möglich }\end{array}$ & $\begin{array}{l}\text { Ausreichend für eine Messstation, Einsatz z. B. als } \\
\text { Server vermutlich zu leistungsschwach }\end{array}$
\end{tabular}

Internetanbindung unumgänglich ist, kann durch entsprechend abgesetzte Antennen eine Verbesserung erzielt werden. Unabhängig davon sind die verfügbaren Übertragungsraten an Extremstandorten meist gering. Es kann daher auch sinnvoll sein, Daten zu komprimieren oder beispielsweise täglich nur einen Datensatz zu übertragen - die weiteren Datensätze befinden sich dann auf einer Speicherkarte. Durch reduzierte Datenübertragung kann auch der Strombedarf gesenkt werden. Andererseits benötigt Datenkomprimierung Rechenaufwand und führt damit ebenfalls zu erhöhtem Stromverbrauch.

Als entscheidende Frage ist auch zu klären, welches Gerät eine Verbindung initiiert. Hier können prinzipiell 2 Varianten unterschieden werden. Diese beiden Varianten werden in Tab. 1 gegenübergestellt:

1. Die Messstation fungiert als Client oder

2. die Messstation fungiert als Server.

Als Lösung für eine integrierte Messstation würde sich auch eine Eigenentwicklung anbieten. In den letzten Jahren wurden die vergleichsweise günstigen Plattformen RaspberryPI und Arduino innerhalb der Internet-Community oft für Messsysteme herangezogen. Hier sind jedoch verschiedene Punkte zu bedenken (siehe Tab. 2).

Zusammenfassend lässt sich sagen, dass ein RaspberryPI auf lange Sicht vermutlich einen $\mathrm{zu}$ hohen Stromverbrauch aufweist, wenngleich er viele
Vorteile bietet (einfache Integration eines Servers, einfaches Multitasking etc.). Demgegenüber bieten Mikrocomputer wie der Arduino eine viel geringere Stromaufnahme, die jedoch mit größerem Entwicklungsaufwand einhergehen. Stellvertretend können diese beiden Plattformen jedoch für eine Vielzahl verschiedener aktueller Plattformen gelten.

Neben der Fragestellung der Plattform ist auch zu klären, welche Sensoren vorhanden sind, und wie diese in ein Messsystem integriert werden können. Da viele auf dem Markt befindliche Messsysteme proprietäre Protokolle verwenden, ist die Auswahl besonders schwierig.

\section{Ausblick optimierte Quellnutzung}

Neben der Datensammlung mittels einer Quellmessstation ist auch die Datenverwertung $\mathrm{zu}$ beachten. Die gesammelten Daten, die sich in der Betriebsleitstelle befinden, bringen keinen Mehrwert, wenn keine weitere Verwertung erfolgt. Es müssen daher vorrangig zwei Ziele verfolgt werden:

- Daten müssen regelmäßig ausgewertet werden, um daraus strategische Entscheidungen ableiten zu können.

- Die Daten müssen unmittelbar automatisiert ausgewertet werden, um betriebliche Entscheidungen treffen zu können.
Die unmittelbare Übergabe der Daten an ein SCADA-System (Supervisory control and data acquisition) ist daher ein logischer Schritt. Somit können auch die 3 Bereiche (Informationsgewinn - Entscheidungen treffen - Aktivität) modular voneinander getrennt betrachtet werden. Daher bietet es sich an, den Informationsgewinn an der Ressource so einfach wie möglich zu gestalten, während ein komplexes (PC-basiertes) System die Entscheidungen trifft und ein Quellmanagement in Kraft tritt. Bei Quellen mit zentraler Stromanbindung kann dieses System auf Basis von Aktuatoren beispielsweise einen Motorschieber betätigen, der eine Quelle ausleitet.

Open access funding provided by University of Natural Resources and Life Sciences Vienna (BOKU).

Open Access Dieser Artikel wird unter der Creative Commons Namensnennung 4.0 International Lizenz (http:// creativecommons.org/licenses/by/4.

$0 /$ deed.de) veröffentlicht, welche die Nutzung, Vervielfältigung, Bearbeitung, Verbreitung und Wiedergabe in jeglichem Medium und Format erlaubt, sofern Sie den/die ursprünglichen $\mathrm{Au}$ tor(en) und die Quelle ordnungsgemäß nennen, einen Link zur Creative Commons Lizenz beifügen und angeben, $o b$ Änderungen vorgenommen wurden. 
ATMEL (2017): Datenblatt Atmega 328, Online im Internet: http://www.atmel.com/images/ Atmel-8271-8-bit-AVR-Microcontroller-ATmega48A48PA-88A-88PA-168A-168PA-328-328P_datasheet Complete pdf [Abruf 1.2.2017]

ELEKTRONIK-KOMPENDIUM (2017): Raspberry Pi: Stromverbrauch messen, Online im Internet: https://www.elektronik-kompendium. de/sites/raspberry-pi/1910071.htm [Abruf 1.2. 2017]

ÖNORM (2001): ÖNORM M 5873-1 - Anlagen zur Desinfektion von Wasser mittels UltraviolettStrahlen - Anforderungen und Prüfung-Anlagen mit Quecksilberdampf-Niederdruckstrahlern BMLFUW (2017): Wasser in Österreich: https:// www.bmlfuw.gv.at/wasser/wasser- oesterreich. html; [Abruf: 17.2.2017]
Triskatis, C. und Tauchmann, H. (2013): Quellfassungsanlagen zur Trinkwasserversorgung Technische und naturwissenschaftliche Grundlagen für den Bau und Betrieb von Quellfassungen für die Wasserversorgung, DIV Deutscher Industrieverlage $\mathrm{GmbH}$ 